\title{
Métodos alternativos de verificação do posicionamento de sondla gástrica em crianças
}

\author{
Alternative methods of verifying the positioning of the gastric tube in children \\ Métodos alternativos de verificación del posicionamiento de la sonda gástrica en niños
}

\begin{abstract}
RESUMO
Objetivo: Identificar os métodos alternativos ao exame radiológico para verificação do posicionamento da sonda gástrica em crianças. Método: Revisão integrativa realizada nas bases de dados LILACS, MEDLINE e Cochrane, de artigos publicados de 2005 a 2015. Os Descritores em Ciências da Saúde (DeCS) utilizados foram: criança e intubação gastrointestinal cruzados por meio dos operadores booleanos AND e OR Resultados: A amostra final foi composta por 5 artigos internacionais, publicados entre 2005 e 2015, todos com delineamento observacional. Os métodos identificados foram: mensuração de pH e bilirrubina, uso do papel tornassol, capnografia, ausculta, borbulhamento de ar, ausência de resíduo gástrico e a características do aspirado. Conclusões: Métodos de verificação não radiológicos em crianças incluem a ausculta gástrica, submersão da sonda em água, uso do papel tornassol, características e incapacidade de obtenção do aspirado, capnografia, mensuração do pH e bilirrubina. No entanto, alguns deles devem ser desencorajados para uso na prática clínica devido as suas limitações, sendo a principal a incapacidade de descartar colocação respiratória.
\end{abstract}

DESCRITORES: Criança; Intubação Gastrointestinal; Segurança do Paciente.

\section{ABSTRACT}

Objective: To identify alternative methods for radiological examination to record the position of the gastric tube in children. Method: Integrative review of the literature performed in the LILACS, MEDLINE and Cochrane databases of articles published from 2005 to 2015. The Health Sciences Descriptors (DeCS) used were: child and gastrointestinal intubation crossed using the booleans operators AND and OR. Results: The final sample consisted of 5 international papers, published between 2005 and 2015, all with an observational design. The methods identified were: measurement of $\mathrm{pH}$ and bilirubin, use of litmus paper, capnography, auscultation, air bubbling, absence of gastric residue and characteristics of the aspirate. Conclusion: Non-radiological verification methods in children include gastric auscultation, immersion of the catheter in water, use of litmus paper, characteristics and inability to obtain the aspirate, capnography, pH measurement and bilirubin. However, some of them should be discouraged for use in clinical practice because of their limitations, the main one being to the inability to rule out respiratory placement.

DESCRIPTORS: Child; Intubation, gastrointestinal; Patient Safety

\section{RESUMEN}

Objetivo: Identificar los métodos alternativos al examen radiológico para verificar el posicionamiento de la sonda gástrica en niños. Método: Revisión integrativa realizada en las bases de datos LILACS, MEDLINE y Cochrane, de artículos publicados entre 2005 y 2015. Los Descriptores de Ciencias de la Salud (DeCS) utilizados fueron: intubación infantil y gastrointestinal cruzada usando los operadores booleanos AND e OR. Resultados: La muestra final fue compuesta por 5 artículos internacionales, publicados entre 2005 y 2015, todos con delineamiento observacional. Los métodos identificados fueron: medición de pH y bilirrubina, uso del papel tornasol, capnografía, auscultación, burbujeación de aire, ausencia de residuo gástrico y características del aspirado. Conclusión: Los métodos de verificación no radiológicos em niños incluyen la auscultación gástrica, sumersión de la sonda en agua, uso del papel tornasol, características e incapacidad de obtención del aspirado, capnografía, medición del pH y bilirrubina. Sin embargo, algunos de ellos deben ser desalentados para su uso en la práctica clínica debido a sus limitaciones, el principal es la imposibilidad de descartar la colocación respiratoria.

DESCRIPTORES: Niño; Intubación Gastrointestinal; Seguridad del Paciente

RECEBIDO EM: 25/08/2020 APROVADO EM: 30/09/2020 


\section{Handria Rodrigues da Silva}

Enfermeira graduada pelo Centro Universitário Ritter dos Reis (UniRitter). Residente em Terapia Intensiva pela Universidade Federal de Ciências da Saúde de Porto Alegre (UFCSPA) com atuação na Irmandade Santa Casa de Misericórdia de Porto Alegre (ISCMPA).

ORCID: 0000-0002-1245-2608

\section{Lívia Pereira Ferreira}

Nutricionista. Pós-graduanda em Nutrição Clinina e Nutrição Materno Infantil pela Estácio de Sá. ORCID: 0000-0002-7822-9855

\section{Morgana Thaís Carollo Fernandes}

Enfermeira. Pós-Doutoranda em Saúde da Criança pela Pontifícia Universidade Católica do Rio Grande do Sul (PUCRS), bolsista pela University of Toronto (UofT). Professora Colaboradora da Residência Multiprofissional em Saúde da Criança e Pesquisadora Associada do Programa de Extensão e Pesquisa em Saúde Urbana, Ambiente e Desigualdades da Universidade Federal do Rio Grande do Sul (UFRGS).

ORCID: 0000-0002-7989-294X

\section{Amanda Pereira Ferreira Dellanhese}

Enfermeira. Doutora em Saúde da Criança e do Adolescente pela Universidade Federal do Rio Grande do Sul. Docente Colaboradora da Residência Integrada Multiprofissional em Saúde Coletiva da Universidade Federal do Rio Grande do Sul.

ORCID: 0000-0002-1515-9693

\section{INTRODUÇÃO}

A inserção de uma sonda gástrica em muitas crianças hospitalizadas usualmente é indicada para suporte nutricional, administração de medicamentos e/ou descompressão gástrica, sendo uma prática privativa do profissional enfermeiro. No entanto, apesar de comum, existem riscos ${ }^{1-2-3}$. Uma sonda mal posicionada pode levar a complicações pulmonares, e em casos mais graves, até a morte ${ }^{4-5}$.

Os dados de prevalência de erro no posicionamento de sondas em crianças relatados na literatura variam e fatores de risco associados à má colocação incluem diminuição do nível de consciência, distensão abdominal, vômitos e sonda orogástrica, além da intubação traqueal ${ }^{1-4-6}$.

O padrão-ouro para verificar a localização da sonda é a radiografia abdominal, mas obter uma imagem radiográfica antes de cada dieta seria caro e impraticável ${ }^{1-5}$. Embora a dose de radiação para um procedimento único pode ser baixa, repetida exposições podem resultar em altas doses cumulativas de radiação, associando-se com vários tipos de câncer, incluindo a leucemia infantil ${ }^{7}$.

Dessa forma, técnicas não radiológicas contribuem para precisão do posicionamento da sonda e diminuição da exposi- ção à radiação em pacientes pediátricos. Este estudo teve como objetivo revisar a literatura para identificar os métodos, alternativos ao exame radiológico, para verificação do posicionamento da sonda gástrica em crianças.

\section{METÓdO}

Trata-se de uma revisão integrativa da literatura, elaborada seguindo a proposta metodológica de Cooper ${ }^{8}$, compreendendo cinco etapas: formulação do problema, coleta de dados, avaliação dos dados, análise e interpretação dos dados e apresentação dos resultados. Este método de pesquisa possibilita reunir e sintetizar o conhecimento já produzido sobre determinado tema ${ }^{8}$.

Para guiar a presente revisão, formulou-se a seguinte questão norteadora: "Em crianças, quais os métodos alternativos ao exame radiológico para verificar o posicionamento da sonda gástrica?"

A busca e seleção dos estudos foi realizada entre os meses de outubro e novembro de 2018, nas bases de dados: Biblioteca Cochrane, Literatura Latino-Americana e do Caribe em Ciências da Saúde (LILACS) e Medical Literature Analysis and Retrieval System On Line (MEDLINE) indexadas na Biblioteca Virtual em Saú- de (BVS). Os Descritores em Ciências da Saúde (DeCS) utilizados foram: criança e intubação gastrointestinal, e seus correspondentes em inglês e espanhol: child e niño, intubation gastrointestinal e intubación gastrointestinal, respectivamente.

O cruzamento entre as versões dos descritores em inglês e espanhol foi realizado utilizando o operador booleano "OR", e entre eles cruzados utilizando-se o operador booleano "AND" nas bases de dados LILACS e MEDLINE. Na busca na Biblioteca Cochrane foi feito o cruzamento dos Medical Subject Headings (MeSH) Child AND Intubation, Gastrointestinal.

Os critérios de inclusão para seleção dos estudos foram: artigos originais, publicados entre 2005 e 2015, disponíveis online na íntegra, gratuitos, nas línguas portuguesa, inglesa e/ou espanhola, que compreendessem técnicas não radiológicas para verificar posicionamento da sonda gástrica em crianças. Foram excluídas monografias, dissertações, teses, textos provenientes de carta ao editor, publicados em periódicos de acesso controlado, duplicados, e aqueles que não respondessem à pergunta norteadora.

Para delimitação da amostra, primeiramente os artigos foram selecionados pelo título e resumo seguindo os critérios de inclusão e exclusão estabelecidos, após 
procedeu-se a leitura na íntegra dos estudos selecionados. Na figura 1 apresenta-se um fluxograma esclarecendo o processo de seleção dos estudos. Para a coleta e avaliação dos dados foi elaborado pela autora um instrumento contendo informações referentes à: título, autores, idioma, país de origem, periódico, ano de publicação, objetivo, desenho metodológico e principais achados.

\section{Figura 1 - Fluxograma do processo de seleção dos artigos}

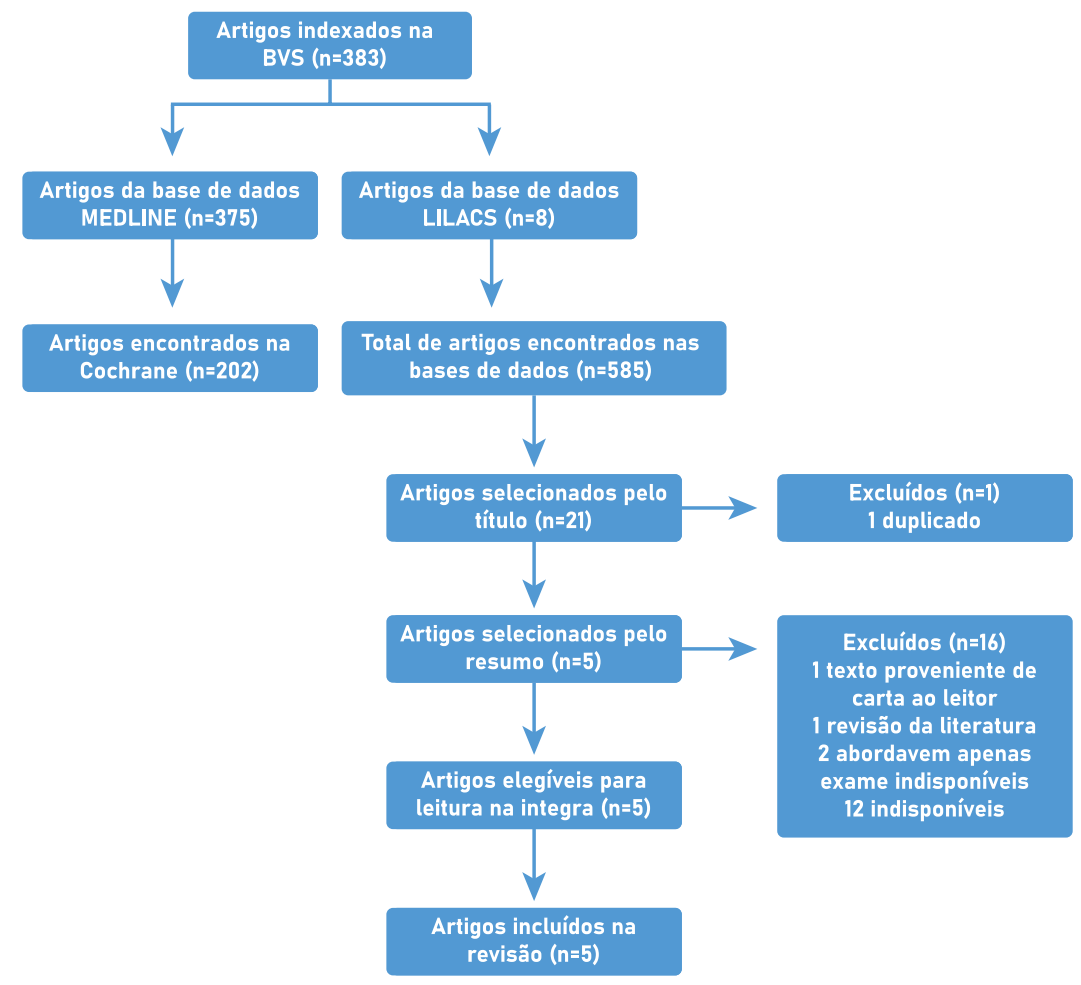

Fonte: Elaborado pelas autoras

\section{Quadro 1 - Sumarização dos Artigos Incluídos na Revisão Integrativa}

\begin{tabular}{|c|c|c|c|c|}
\hline $\mathbf{N}^{\circ}$ & AUTORES, TÍTULO & ANO & PERIÓDICO & PAÍs \\
\hline 1 & $\begin{array}{l}\text { Ellet MLC, Croffie JMB, Cohen MD, Perkins, SM }{ }^{9} \\
\text { Gastric tube placement in young chil-dren }\end{array}$ & 2005 & Clinical Nursing Research & Estados Unidos \\
\hline 2 & $\begin{array}{c}\text { Freer } \mathrm{Y}, \text { Lyon } \mathrm{A}^{10} \\
\text { Risk management, or just a different risk? }\end{array}$ & 2006 & $\begin{array}{l}\text { Archieves of Disease in Childhood: } \\
\text { Fetal and Neonatal Edition }\end{array}$ & Inglaterra \\
\hline 3 & $\begin{array}{c}\text { Gilbertson HR, Rogers EJ, Ukoumunne } \mathrm{OC}^{11} \\
\text { Determination of a practical pH cutoff level for reliable confir- } \\
\text { mation of nasogastric tube placement }\end{array}$ & 2011 & $\begin{array}{l}\text { Journal of Par-enteral and En-teral } \\
\text { Nutrition }\end{array}$ & Estados Unidos \\
\hline 4 & $\begin{array}{l}\text { Ellet, MLC, Cohen MD, Croffie JMB, Lane KA, Austin JK, Perkins } \\
\qquad \text { SM }^{12} \\
\text { Comparing bedside methods of deter-mining placement of } \\
\text { gastric tubes in Children }\end{array}$ & 2014 & $\begin{array}{l}\text { Journal for Spe-cialists in Pedi-atric } \\
\text { Nursing }\end{array}$ & Estados Unidos \\
\hline 5 & $\begin{array}{l}\text { Meert KL, Caverly M, Kelm LM, Metheny NA }{ }^{13} \\
\text { The pH of feeding tube aspirates from critically ill infants }\end{array}$ & 2015 & American Jour-nal of Critical Care & Estados Unidos \\
\hline
\end{tabular}




\section{artigo}

Rodrigues da Silva, H.; Ferreira, L.P.; Fernandes, M.T.C.; Dellanhese, A.P.F.

Métodos alternativos de verificação do posicionamento de sonda gástrica em crianças

\section{Quadro 2 - Síntese dos Principais Achados dos Estudos}

\begin{tabular}{|c|c|c|c|c|}
\hline $\mathbf{N}^{\circ}$ & DESENHO & AMOSTRA & TÉCNICAS INVESTIGADAS & PRINCIPAIS ACHADOS \\
\hline $1^{9}$ & Observacional & $\begin{array}{c}72 \text { crianças até } 7 \\
\text { anos de idade }\end{array}$ & $\begin{array}{c}\mathrm{pH} \leq 5, \text { bilirrubina } \geq 5 \mathrm{mg} / \mathrm{dl} \\
\text { e capnografia comparado ao } \\
\text { exame radiológico }\end{array}$ & $\begin{array}{l}\text { Posicionamento correto em 79,2\% ( } 57 / 72 \text { ) validados } \\
\text { pelo exame radiológico. Utilizando o limite sugerido de } \\
\text { pH foram identificadas } 34 / 40 \text { (VPN } 85 \% \text { ) das crianças } \\
\text { com sonda em posicionamento gástrico. Não houve } \\
\text { diferença estatística entre pH e frequência alimentar } \\
\text { ou uso de inibidores de secreção gástrica. O sugerido } \\
\text { corte de bilirrubina } \geq 5 \mathrm{mg} / \mathrm{dl} \text { não identificou nenhuma } \\
\text { das duas sondas posicionadas incorretamente, ambas } \\
\text { com valores abaixo, mas posicionadas no duodeno. Não } \\
\text { foram detectadas sonda no trato respiratório, níveis de } \\
\text { cO2 foram de } 0-2 \mathrm{mmHg} \text {, abaixo do corte estabelecido } \\
\text { em pacientes adultos < } 15 \mathrm{mmHg} \text {. }\end{array}$ \\
\hline
\end{tabular}

Dos 207 questionários enviados, houve 165 (80\%) respostas. $55 \%$ das unidades usavam o papel tornassol e $45 \%$ a fita de $\mathrm{pH}$. Entretanto, todas as unidades usaram testes complementares se os resultados fossem inconclusivos ou se ausência de resíduo gástrico. Dentre estes testes estavam a ausculta e as características do aspirado, além de uma unidade reportar o uso de "borbulhamento de ar" para identificar sonda posicionada em vias aéreas. Houve uma variação considerável no valor de $\mathrm{pH}$ seguro para alimentar.

Um pH $\leq 4$ como ponto de corte para assumir posicionamento gástrico é impraticável, um valor de $\mathrm{pH} \leq 5$ seria mais apropriado. $\mathrm{O}$ valor de $\mathrm{pH}$ do aspirado endotraqueal foi de 6 . Uma sonda posicionada no esôfago foi identificada com pH de 5.5. Sugere-se realizar exame radiográfico se valor do $\mathrm{pH}>5$. A coloração do aspirado foi relatada como um método de confirmação da sonda quando $\mathrm{pH}>4$. As cores esbranquiçada, clara e verde podem indicar localização gástrica.

\begin{tabular}{l|l|c|c}
$3^{11}$ & Observacional & $\begin{array}{c}664 \text { crianças até } 5 \\
\text { anos de idade }\end{array}$ & $\begin{array}{c}\text { Teste pH gástrico e endotra- } \\
\text { queal }\end{array}$ \\
$4^{12}$ & Observacional & $\begin{array}{c}276 \text { crianças até 17 } \\
\text { anos de idade (173 } \\
\text { recém-nascidas) }\end{array}$ & $\begin{array}{l}\text { pH (limítrofe } 5 \text { para crianças em } \\
\text { jejum e 6 para crianças alimen- } \\
\text { tadas), bilirrubina e capnografia }\end{array}$ \\
\hline $5^{13}$ & Observacional & 54 recém-nascidas & $\begin{array}{l}\text { Teste pH em situações com e } \\
\text { sem uso inibidores da secreção } \\
\text { gástrica, em crianças em jejum } \\
\text { e alimentadas }\end{array}$ \\
\hline
\end{tabular}

Embora usar o $\mathrm{pH}$ para detectar posicionamento no estômago varia de $87 \%$ a 92,2\% (especificidade), o método mais efetivo para detectar erro na localização é a ausência de resíduo gástrico (VPP 66,7\%). As cores branca, verde e bronze podem indicar a localização gástrica da sonda. Entretanto, a consistência do aspirado não se mostrou útil para avaliação do posicionamento. Não foi possivel avaliar bilirrubina e $\mathrm{CO}$, pois não apresentaram variabilidade.

Independente do uso de inibidores da secreção gástrica e se a criança estava ou não em jejum, 0 aspirado muitas vezes tem valor de $\mathrm{pH} \leq 5.5 \mathrm{em} 90 \%$ dos casos em que a sonda estava corretamente posicionada na avaliação radiológica. 
cas à beira leito que garantam a segurança dos pacientes, uma vez que, o uso rotineiro de confirmação radiográfica não é uma prática adequada antes de cada alimentação ou administração de medicamentos ${ }^{5}$.

A ausculta gástrica é o método que tradicionalmente é utilizado para avaliar a localização da sonda em pacientes pediátricos, todavia não há evidências de sua efetividade em descartar colocação respiratória ${ }^{1-5}$. Um dos estudos ${ }^{10}$ revisados evidenciou que na incapacidade de obtenção de aspirado, os enfermeiros utilizam este método como teste confirmatório para posicionamento gástrico. Entretanto, inflar ar nas vias aéreas produz um som indistinguível do ar inflado no trato gastrointestinal.

A observação de borbulhamento de ar, também relatada neste estudo ${ }^{10}$, é um método pouco confiável, pois o estômago pode conter ar e indicar falsamente a colocação respiratória ${ }^{1-4}$. Além disso, o método de submersão pode aumentar o risco de aspiração com a inspiração do paciente4.

Nos estudos que observaram testes de acurácia para posicionamento incorreto da sonda $a^{9-12}$, a ausência de aspirado teve o indicador mais elevado de valor preditivo positivo (VPP 66,7\%), este valor representa assertivamente o posicionamento incorreto. Entretanto, a literatura evidencia que a incapacidade de obtenção de aspirado não indica necessariamente que a sonda esteja mal posicionada, pois pode haver presença de conteúdo estomacal marginal, ou que a ponta da mesma não esteja em contato com os fluidos gástricos ${ }^{14}$

A avaliação de bilirrubina não se mostrou um indicador confiável, pois não identificou as sondas posicionadas no intestino ${ }^{9}$. A concentração desta substância pode variar dependendo da localização da sonda, resíduo com nível de bilirrubina de $\geq 5 \mathrm{mg} / \mathrm{dl}$ tem sido associado a posicionamento intestinal em pacientes adultos, entretanto há divergências na literatura acerca desta concentração em crianças ${ }^{5}$.

O método mais confiável para confirmar a colocação da sonda gástrica é a mensuração do $\mathrm{pH}$ do resíduo aspirado ${ }^{1}$. O estudo que investigou a acurácia para sonda corretamente posicionada demonstrou

\section{A avaliação}

de bilirrubina

não se mostrou

um indicador

confiável, pois

não identificou as

sondas posicionadas

no intestino. A

concentração desta

substância pode

variar dependendo da

localização da sonda,

resíduo com nível de

bilirrubina de $\geq 5 \mathrm{mg}$ /

dl tem sido associado

a posicionamento

intestinal em

pacientes adultos,

entretanto há

divergências na

literatura acerca

desta concentração

em crianças. que a utilização do $\mathrm{pH}$ tem valor preditivo negativo (VPN) de $85 \%$, este valor representa assertivamente o posicionamento correto da sonda?.

A utilização de inibidor de secreção gástrica, assim como a frequência alimentar, levanta questionamentos acerca da segurança deste teste, uma vez que teriam potencial para elevar o $\mathrm{pH}$ gástrico ${ }^{1-4}$. No entanto, os estudos que compararam o $\mathrm{pH}$ estomacal, não encontraram diferenças significativas entre as crianças que recebiam e não recebiam estes medicamentos, bem como entre as quais eram alimentadas de maneira contínua, intermitente ou que estavam em jejum ${ }^{12-13}$.

Apesar de não haver um consenso estabelecido na literatura, os estudos demonstraram que o valor de $\mathrm{pH} \leq 5$ é um bom preditor para localização gástrica ${ }^{9-11-12}$. Secreções brônquicas e esofágicas comumente têm $\mathrm{pH}>6$, embora o $\mathrm{pH}$ esofágico possa ser ácido na presença de refluxo gástrico ${ }^{1}$.

$\mathrm{O}$ pH superior a 5 não prevê de maneira confiável a inserção correta da sonda, podendo indicar inserção respiratória ou esofágica ${ }^{15}$, um caso de $\mathrm{pH} 5.5$ em posição esofágica foi reportado em um dos estudos incluídos nesta revisão ${ }^{11}$.

Um dos estudos apontou o uso do papel tornassol como um método de verificação da sonda ${ }^{10}$. É defendido que este método acidez/alcalinidade seja substituído, pois tem baixa sensibilidade em distinguir entre secreções gástricas e brônquicas, em especial o papel tornassol azul ${ }^{1-16}$.

O papel tornassol azul ficará rosa na presença de um meio ácido, independentemente do nível de acidez, isto é, valor de $\mathrm{pH}$ inferior a 7 , por isso não é suficientemente sensível para distinguir entre o $\mathrm{pH}$ dos fluidos. É possível que uma sonda mal posicionada não seja detectada, um incidente foi relatado onde isso contribuiu para a morte do paciente ${ }^{16}$.

Alguns pesquisadores relatam que associar a mensuração de $\mathrm{pH}$ e coloração do aspirado melhoram o sucesso em prever a colocação correta da sonda, uma vez que o aspirado possui coloração específica para o local de colocação pretendi$\mathrm{do}^{4-5}$. A coloração branca, verde e bronze 
foi indicativo de colocação gástrica nos estudos revisados ${ }^{11}$, embora a consistência não tenha sido útil ${ }^{12}$.

A utilização da capnografia mostra-se promissora para identificar extravio pulmonar, uma vez que a ausência de sinais de desconforto respiratório pode ser ineficaz na detecção de uma sonda mal posicionada, especialmente em pacientes inconscientes e intubados1, embora não seja útil para discernir diferenças no posicionamento esofágico, gástrico ou intestinal ${ }^{4-5}$. Em crianças, conforme estudo revisado, nenhuma sonda estava no trato respiratório, ainda que nem todas estivessem no estômago?. Os valores da capnografia foram $0-2 \mathrm{mmHg}$, bem abaixo do limite estabelecido para pacientes adultos, $\leq 15 \mathrm{mmHg}$.

Há evidências de diversos métodos não radiológicos usados para verificação do posicionamento da sonda gástrica em crianças. Entretanto, para uso na prática clínica devem-se considerar as limitações dos métodos garantindo a segurança do paciente.

\section{CONCLUSÕES}

Em vista dos possíveis resultados catastróficos que podem ocorrer por uma sonda mal posicionada, é imperativo a verificação contínua do posicionamento da sonda em crianças. Métodos de verificação não radiológicos em crianças incluem a ausculta gástrica, submersão da sonda em água, uso do papel tornassol, características e incapacidade de obtenção do aspirado, capnografia, mensuração do $\mathrm{pH}$ e bilirrubina.

A ausculta, submersão da sonda e o uso do papel tornassol, em especial o azul, devem ser desencorajados para uso na prática clínica. $\mathrm{O}$ método mais acurado para avaliar a localização da sonda é o teste de $\mathrm{pH}$, um nível de 5 ou inferior sugere posicionamento gástrico. Os resultados desta revisão foram limitados pela escassez de pesquisas com foco em crianças, e a predominância de estudos observacionais sugere a necessidade de novos estudos que elevem o nível de evidência das práticas de enfermagem.

\section{REFERÊNCIAS}

1. Peter S, Gill F. Development of a clinical practice guideline for testing nasogastric tube placement. J Spec Pediatr Nurs. 2009;14(1):3-11.

2. Vincent CA, Lee ACH, Hanna GB. Patient safety alerts: a balance between evidence and action. Arch Dis Child Fetal Neonatal Ed. 2006;91(5):314-315.

3. Conselho Federal de Enfermagem. Resolução n 453, de 16 de janeiro de 2014. Aprova a norma técnica que dispõe sobre a atuação da equipe de enfermagem em terapia nutricional.

4. Irving AY, Lyman B, Northington L, Bartlett JA, Kemper C. Nasogastric tube placement and verification in children: review of the current literature. Critic Care Nurse. 2014;34(3):67-78.

5. Makic MBF, Rauen C, Watson R, Poteet AW. Examining the evidence to guide practice: challenging practice habits. Critic Care Nurse. 2014;34(2):28-45.

6. Ellet ML, Maahs J, Forsee S. Prevalence of feeding tube placement errors and associated risk factors in children. MCN Am J Matern Child Nurs. 1998;23(5):234-9.

7. Kleinerman RA. Cancer risk following diagnostic and therapheutic radiation exposure in children. Pediatr Radiol. 2006;36(2):121-25.

8. Cooper HM. Scientific guidelines for conducting integrative research reviews. Review of Educational Research Summer. 1982;52(2):291-302.

9. Ellet MLC, Croffie JMB, Cohen MD, Perkins SM. Gastric tube placement in young children. Clin Nurs Res. 2005;14(3):238-52.

10. Freer R, Lyon A. Risk management, or just a different risk? Arch Dis Child Fetal Neonatal Ed. 2006;91(5):327-9.

11. Gilbertson HR, Rogers EJ, Ukoumunne OC. Determination of a practical $\mathrm{pH}$ cutoff level for reliable confirmation of nasogastric tube placement. JPEN J Parenter Nutr. 2011;35(4):540:4.

12. Ellet MLC, Cohen MD, Croffie JMB, Lane KA, Austin JK, Perkins $\mathrm{SM}$. Comparing bedside methods of determining placement of gastric tubes in children. J Spec Pediatr Nurs. 2014;19(1):68-79.

13. Meert KL, Caverly M, Kelm LM, Metheny NA. The pH of feeding tube aspirates from critically III infants. Am J Crit Care. 2015;24(5):e72-2.

14. Bowden VR, Greenberg CS. Procedimentos de enfermagem pediátrica. 3 ed. Rio de Janeiro: Guanabara Koogan, 2013. p. 228-235.

15. Hockenberry MJ, Wilson D. Fundamentos de enfermagem pediátrica. 9 ed. Rio de Janeiro: Elsevier, 2014. p. 610-76

16. NHS Scotland. Nasogastric feeding tubes: undetected maplposition. Disponivel em: www.hfs.scot.nhs.uk/publications/1482171667-PSAN0428.pdf 